\title{
A new allergic rhinitis therapy (MP29-02*) provides nasal and ocular symptom relief days faster than current firstline monotherapies
}

\author{
Peter Hellings ${ }^{1 *}$, Claus Bachert ${ }^{2}$, Ralph Mösges ${ }^{3}$, Glenis Scadding ${ }^{4}$, Ullrich Munzel ${ }^{5}$, Wytske Fokkens ${ }^{6}$ \\ From The 10th Symposium of Experimental Rhinology and Immunology of the Nose (SERIN 2015) \\ Stockholm, Sweden. 19-21 February 2015
}

\section{Background}

The efficacy of MP29-02* (a novel intranasal formulation of azelastine hydrochloride [AZE] and fluticasone propionate $[\mathrm{FP}]$ in an advanced delivery system) in providing overall nasal and ocular symptom relief vs AZE, FP or placebo (PLA) has been assessed.

\section{Methods}

Six hundred and ten moderate-to-severe SAR patients ( $\geq 12$ yrs) were randomized into a double-blind, PLAcontrolled, 14-day, parallel-group, trial (NCT00660517) to MP29-02*, AZE, FP or PLA nasal sprays (1 spray/ nostril bd; daily dose: $\mathrm{AZE}=548 \mu \mathrm{g} ; \mathrm{FP}=200 \mu \mathrm{g}$ ) [1]. Change from baseline (CFB) in reflective total of 7 symptom scores (rT7SS; AM + PM, max=42) was assessed post-hoc. CFB in rT7SS and each nasal (congestion, itching, rhinorrhea, sneezing) and ocular symptom (itching, redness, watering; $\max =6$ each) was assessed over time.

\section{Results}

Overall, MP29-02* patients showed greater reduction in rT7SS vs FP, AZE \& PLA (relative diff: $52 \%$ to FP $(\mathrm{p}=0.0013), 56 \%$ to AZE $(\mathrm{p}=0.0004))$ evident from treatment day 1 vs FP $(\mathrm{p}=0.0072)$, AZE $(\mathrm{p}=0.0336)$ or PLA $(\mathrm{p}<0.0001)$ and sustained for 14 days. The level of relief achieved by MP29-02* patients on Day $2(-5.52)$ was not achieved before Day 5 by FP patients or Day 8 by AZE patients. This pattern of rapid, sustained and superior symptom relief by MP29-02* was observed for each nasal and ocular symptom, which was not the case with FP and AZE. MP29-02* provided significantly superior nasal congestion relief than FP or AZE from Day 2; the

${ }^{1}$ University Hospitals Leuven, Dept of Otorhinolaryngology, Head \& Neck Surgery, Leuven, Belgium

Full list of author information is available at the end of the article level of congestion relief provided by MP29-02* on Day 2 $(-0.77)$ was not achieved before Day 6 and Day 9 for FP and AZE, respectively. MP29-02* provided significantly superior ocular itching relief vs FP from Day 2 and vs AZE from Day 3; the ocular itch relief provided by MP29-02* on Day $2(-0.77)$ was not achieved before Day 10 for FP-patients. Similarly, the level of ocular itch relief provided by MP29-02* on Day $3(-1.06)$ was not achieved by AZE-patients before Day 11 .

\section{Conclusion}

The consistent and rapid effect in alleviating all nasal and ocular symptoms is unique to MP29-02* and contributes to its superiority over AZE and FP. The time advantage over firstline therapy in achieving significant relief and sustained effect should improve patient concordance. MP29-02* is considered a new standard of care in AR.

* Dymista

\section{Authors' details}

'University Hospitals Leuven, Dept of Otorhinolaryngology, Head \& Neck Surgery, Leuven, Belgium. ${ }^{2}$ Ghent University Hospital, Department of OtoRhinolaryngology, Ghent, Belgium. ${ }^{3}$ University of Cologne, IMSIE, Cologne, Germany. ${ }^{4}$ The Royal National Throat Nose and Ear Hospital, London, UK. ${ }^{5}$ Meda, Corporate Clinical Affairs, Bad Homburg, Germany. ${ }^{6}$ Academic Medical Center, Department of Otorhinolaryngology, Amsterdam, Netherlands.

Published: 26 June 2015

Reference

1. Meltzer E, Ratner P, Bachert C, Carr W, Berger W, Canonica GW, et al: . Int Arch Allergy Immunol 2013, 161(4):369-77.

\section{doi:10.1186/2045-7022-5-S4-P34}

Cite this article as: Hellings et al:: A new allergic rhinitis therapy (MP29$\left.02^{*}\right)$ provides nasal and ocular symptom relief days faster than current firstline monotherapies. Clinical and Translational Allergy 2015 5(Suppl 4):P34. 